\title{
Plasmablastic lymphoma presenting with ileocecal intussusception in an immunocompetent patient
}

Ok Ran Shin ${ }^{1}$, Jiyeon Hyeon ${ }^{2}$, Sang-Bum Kang ${ }^{3}$, and Jong Ok Kim ${ }^{4}$

${ }^{1}$ Department of Pathology, Uijeongbu St. Mary's Hospital, College of Medicine, The Catholic University of Korea, Uijeongbu; ${ }^{2}$ Department of Pathology, Incheon St. Mary's Hospital, College of Medicine, The Catholic University of Korea, Incheon; Departments of ${ }^{3}$ Internal Medicine and ${ }^{4}$ Pathology, Daejeon St. Mary's Hospital, College of Medicine, The Catholic University of Korea, Daejeon, Korea
Received: November 13, 2018 Revised : December 19, 2018 Accepted: December 19, 2018

\section{Correspondence to} Jong Ok Kim, M.D.

Tel: +82-42-220-9611

Fax: $+82-42-220-9843$

E-mail:jkim@catholic.ac.kr
A 25-year-old man was referred to our hospital with melena and low abdominal pain. Abdominal computed tomography showed an 8-cm enhancing mass near the ileocecal valve leading to intussusception (Fig. 1A). Colonoscopy identified a huge ulcerated mass pro- truding into the proximal ascending colon (Fig. 1B). Pathological examination revealed the proliferation of diffuse large discohesive polygonal cells having high nuclear/cytoplasmic ratio which were $\mathrm{CD}_{13} 8-, \mathrm{CD}_{3} 8-$, and Ki-67(high expression, 90\%) positive; and
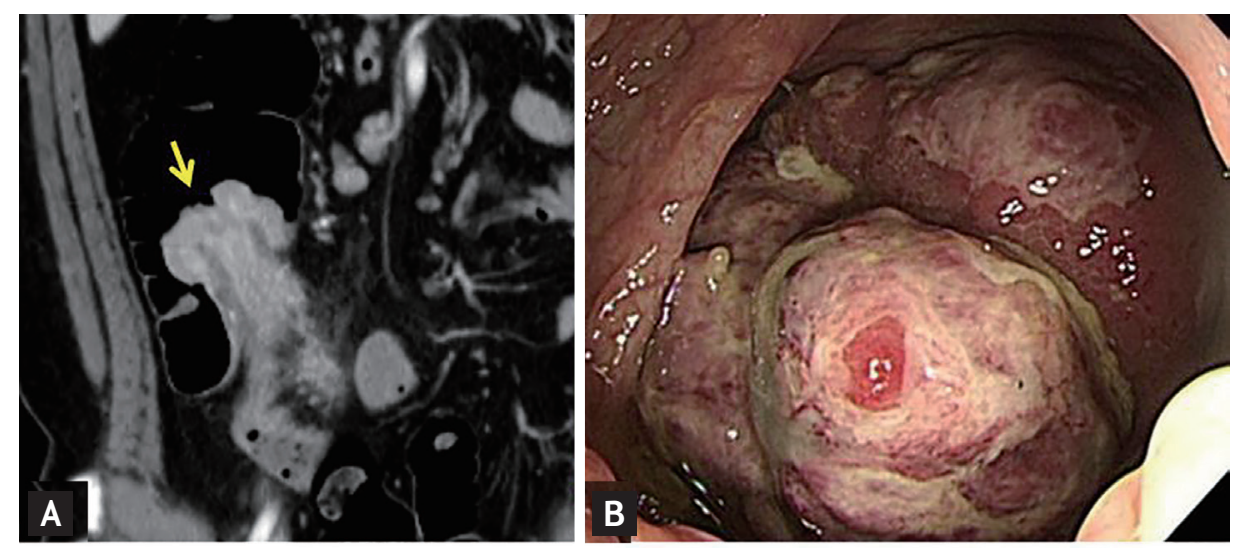

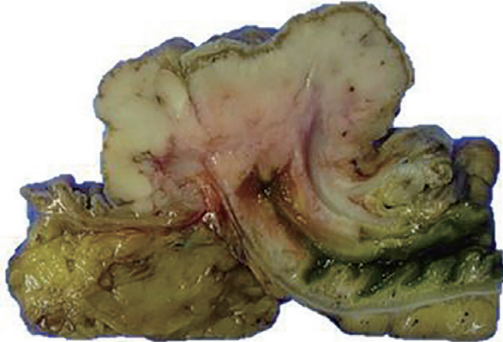

C

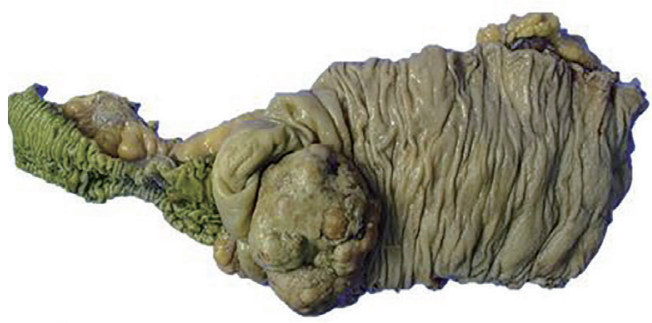

D

Figure 1. (A) Ileocecal intussusception is detected on the coronal view of the abdominal and pelvic computed tomography with enhancement. A large enhancing leading point mass (arrow), thickened walls of the intussuscipiens, mesenteric fat and vessels are seen. Also, multiple enhancing ileocolic lymph nodes are noted. (B) A huge fungating mass with ulceration protrudes into the proximal ascending colon on the colonoscopic finding. (C) The cut surface of the ileocecal mass shows intussusception into the ascending colon. (D) A hemicolectomy specimen shows an $8 \times 6-\mathrm{cm}$ fungating mass arising from the ileocecal valve. 

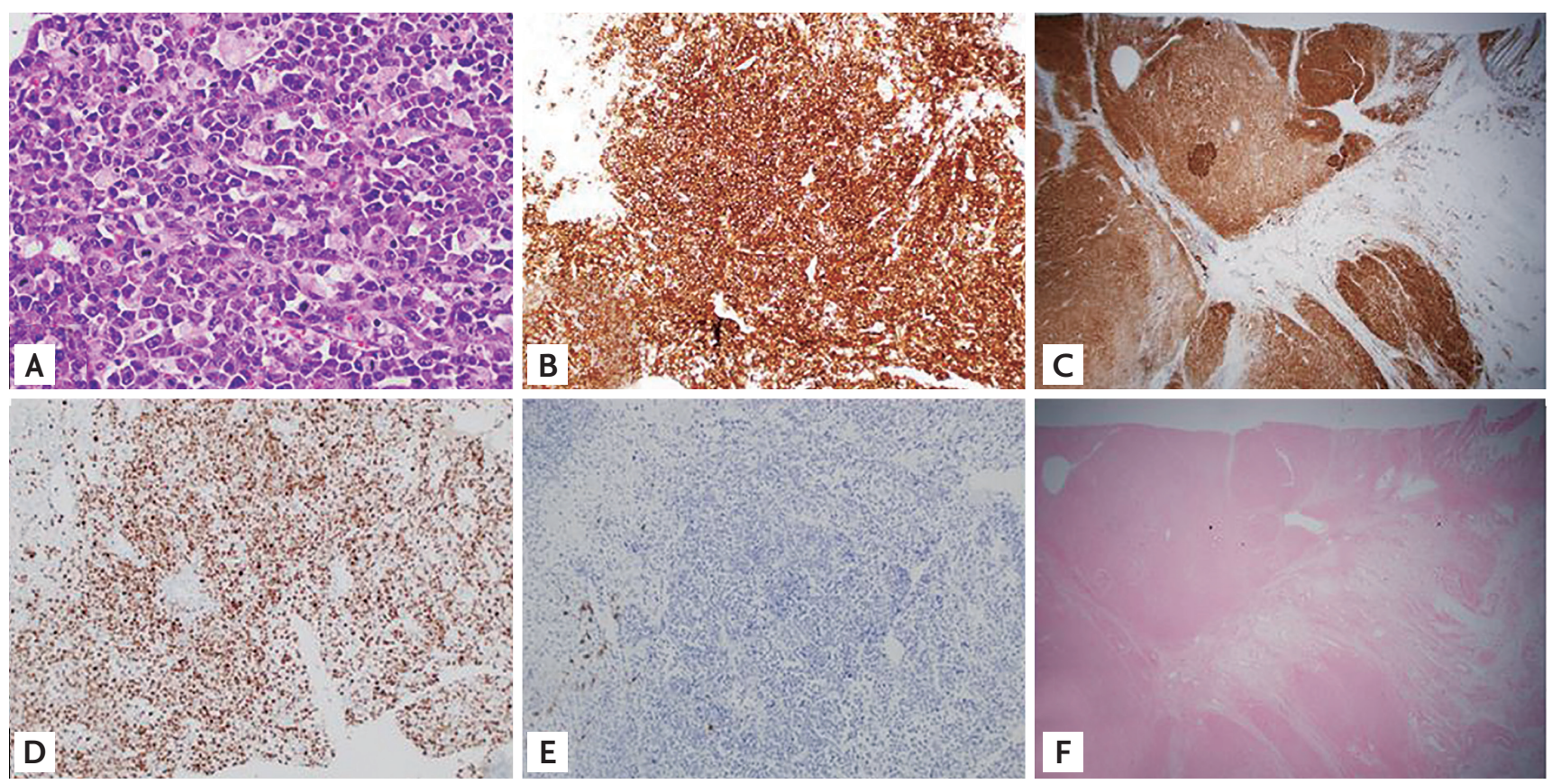

Figure 2. Microscopic findings of plasmablastic lymphoma (PBL) with plasmacytic differentiation. (A) Diffuse proliferation of large discohesive polygonal cells with a high nuclear/cytoplasmic ratio, eccentric nuclei, prominent central nucleoli, and a moderate amount of basophilic cytoplasm (H\&E, $\times 100)$; (B) PBL cells were immunohistochemically positive for CD 138 ( $\times 40)$; (C) positive for CD 38 (×10); (D) positive for Ki-67 (90\%) (×40); (E) negative CD 20 (×40); (F) in situ hybridization for Epstein-Barr virus-encoded small RNA showed negative expression of all PBL cells $(\times 10)$.

CD20-, CD3-, and cytokeratin-negative. Plasma cell neoplasm or plasmablastic lymphoma (PBL) was suspected. Serum and urine protein electrophoresis results were unremarkable. A right hemicolectomy was performed owing to risk of impending intestinal obstruction. The specimen showed an $8-\times 6$-cm fungating mass arising from the ileocecal valve. The cut surface of the mass showed intussusception into the ascending colon (Fig. ${ }_{1} \mathrm{C}$ and ${ }_{1 D} \mathrm{D}$ ). The resected tumor cells were identical to the endoscopic biopsy cells and spread from the mucosa to the serosa (Fig. 2A-2E). The results were negative for serum human immunodeficiency virus (HIV) and Epstein-Barr virus (EBV) in situ hybridization expression (Fig. 2F). The final diagnosis was PBL with plasmacytic differentiation. The patient is in remission after six EPOCH (etoposide, prednisolone, oncovin, cyclophosphamide, hydroxydaunorubicin) chemotherapy cycles.

PBL is a rare form of non-Hodgkin's lymphoma associated with HIV infection, which shows strong affinity for the oral cavity. EBV seems to play an important role in HIV-associated PBL oncogenesis. However, PBL is not strongly associated with HIV or EBV infection in Koreans, and it shows variable organ distribution without oronasal predilection. We report a rare case of PBL involving the ileocecal valve in an HIV/EBV-negative immunocompetent patient presenting with intussusception into the ascending colon. Clinicians might not suspect PBL in HIV- and EBV-negative immunocompetent patients with extraoral lesions. Clinician and pathologist awareness of such unusual presentation is necessary for early diagnosis, appropriate treatment, and improved outcomes.

The case was approved by the Institutional Review Board of Daejeon St. Mary Hospital, College of Medicine, The Catholic University of Korea (IRB No. DC18ZESE0112). Informed consent was confirmed by the IRB.

\section{Conflict of interest}

No potential conflict of interest relevant to this article was reported. 